\title{
Propionate metabolism in Saccharomyces cerevisiae: implications for the metabolon hypothesis
}

\author{
Jack T. Pronk, Annelies van der Linden-Beuman, Cornelis Verduyn, $\dagger$ \\ W. Alexander Scheffers and Johannes P. van Dijken
}

Author for correspondence: Jack T. Pronk. Tel: +31 15 782387. Fax +31 15782355.

Department of Microbiology and Enzymology,

Kluyver Laboratory of

Biotechnology, Delft

University of Technology,

Julianalaan 67, 2628 BC

Delft, The Netherlands
Aerobic, glucose-limited chemostat of Saccharomyces cerevisiae CBS 8066 cometabolized propionate when this compound was added to the reservoir medium. Co-metabolism of propionate led to an increase of the biomass and protein yields. Attempts to grow $S$. cerevisiae on propionate as a sole source of carbon and energy were not successful. Activities of propionyl-CoA synthetase in cell-free extracts were sufficient to account for the rates of propionate consumption observed in the chemostat cultures. Activities of propionyl-CoA carboxylase, a key enzyme of the methylmalonyl-CoA pathway of propionate metabolism, were negligible. In contrast, activities of 2methylcitrate synthase, a key enzyme activity of the 2-methylcitrate pathway of propionate metabolism, increased substantially with increasing propionateto-glucose ratios in the reservoir media, and were sufficient to account for the propionate consumption rates observed in the chemostat cultures. This suggested that the 2-methylcitrate pathway is the major pathway of propionate metabolism in S. cerevisiae. In the literature, labelling patterns observed after incubation of this yeast with $\left[3-{ }^{-13} \mathrm{C}\right]$ propionate have been interpreted as evidence for channelling of tricarboxylic acid (TCA) cycle intermediates, possibly as a consequence of the organization of TCA cycle enzymes in a metabolon. However, this interpretation of ${ }^{13} \mathrm{C}$-labelling patterns rested on the assumption that propionate metabolism in S. cerevisiae occurs via the methylmalonyl-CoA pathway. Since the distribution of ${ }^{13} \mathrm{C}$ in alanine reported in the literature is fully compatible with a major role of the 2-methylcitrate pathway in propionate metabolism, it cannot be interpreted as evidence for the existence of a TCA cycle metabolon in S. cerevisiae.

Keywords: Saccharomyces cerevisiae, propionate metabolism, 2-methylcitrate pathway, metabolic channelling

\section{INTRODUCTION}

Non-metabolizable weak acids such as benzoate, butyrate and, under anaerobic conditions, acetate, have a profound effect on the physiology of yeasts. When these acids are added to the medium feed of sugar-limited chemostat cultures, the biomass yield decreases and the rate of sugar dissimilation increases (Verduyn et al., 1990a, b; 1992). This negative effect on growth efficiency can be attributed to an increased energy requirement for cytosolic $\mathrm{pH}$

†Present address: BIRD Engineering, PO Box 149, 3100 AC Schiedam, The Netherlands. homeostasis by the proton-pumping plasma membrane ATPase.

As anticipated, the addition of propionate to anaerobic, glucose-limited chemostat cultures of $S$. cerevisiae also caused a decreased biomass yield and an increased rate of alcoholic fermentation (Verduyn et al., 1990a, b). However, when low concentrations $(5-10 \mathrm{mM}$ ) of propionate were added to aerobic glucose-limited chemostat cultures of $S$. cerevisiae growing at low dilution rates, virtually all propionate disappeared from the cultures (C. Verduyn, unpublished observations).

In a variety of prokaryotic organisms, propionate is metabolized by the methylmalonyl-CoA pathway of 
(a)

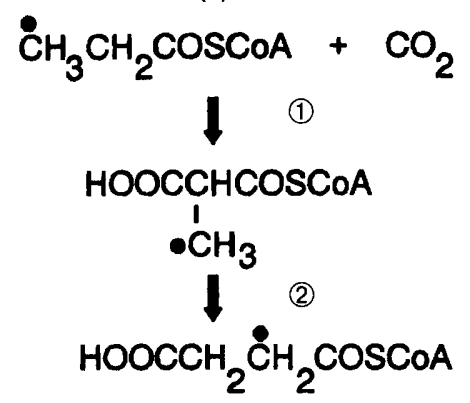

(3)

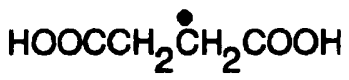

${ }_{\mathrm{HOOCCHOHCH}} \mathrm{COOH}$

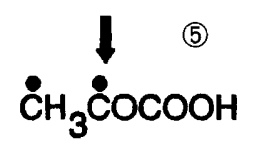

(b)
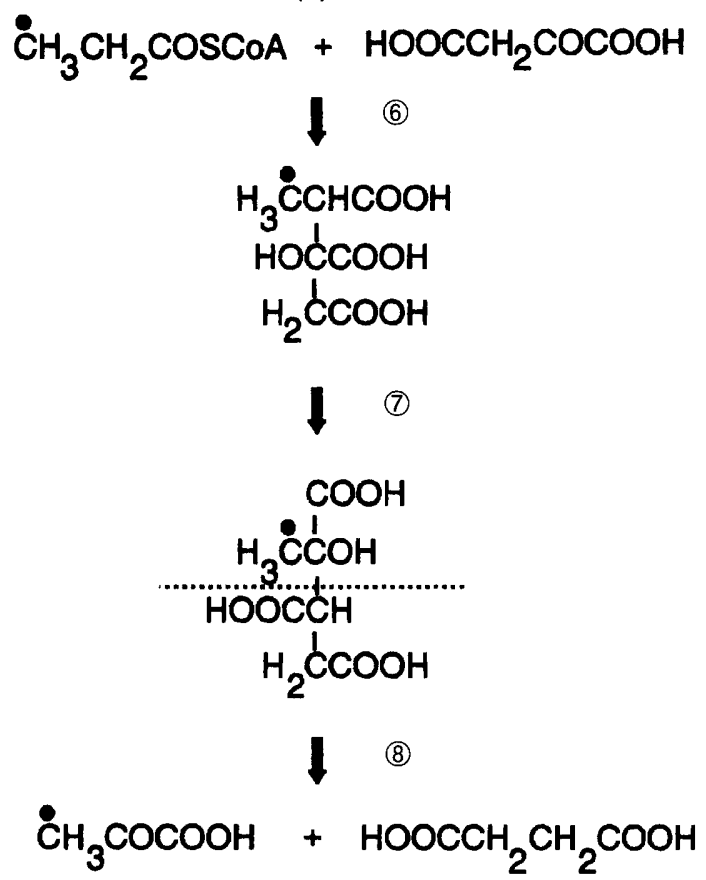

Fig. 1. Pathways of propionate metabolism. (a) Methylmalonyl-CoA pathway; (b) 2-methylcitrate pathway. Black dots indicate the fate of the C-3 atom of propionate when propionate is converted to pyruvate (or alanine), assuming that all intermediates are in equilibrium with the bulk phase water and that pyruvate does not re-enter the TCA cycle. Numbered reactions are catalysed by the following enzymes: 1, propionyl-CoA carboxylase; 2, methylmalonyl-CoA mutase; 3, succinyl thiokinase; 4, TCA cycle enzymes; 5, malate dehydrogenase, phosphoenolpyruvate carboxykinase and pyruvate kinase; 6, 2-methylcitrate synthase; 7, 2-methylcitrate dehydratase and 2-methylisocitrate dehydratase; 8, 2-methylisocitrate lyase.

propionate metabolism (Fig. 1a; Wegener et al., 1968). However, we have found no evidence in the literature to indicate the involvement of this route in propionate metabolism in yeasts. One of the key enzymes of this pathway, methylmalonyl-CoA mutase, requires vitamin B12 as a cofactor (Wegener $e t$ al., 1968), and S. cerevisiae is not known to be able to synthesize this vitamin (Schneider \& Stroinski, 1987). An alternative pathway of propionate metabolism has been demonstrated in the lipid-degrading yeast Yarrowia lipolytica (Tabuchi \& Uchiyama, 1975; Uchiyama et al., 1982). This pathway is initiated by the synthesis of 2-methylcitrate from propionyl-CoA and oxaloacetate (Fig. 1b). Via a metabolic sequence similar to the 'normal' TCA cycle reactions, 2-methylcitrate is converted to 2-methylisocitrate, which is then cleaved into pyruvate and succinate (Fig. $1 \mathrm{~b}$ ).

Metabolism of $\left[3-{ }^{13} \mathrm{C}\right]$ propionate by $S$. cerevisiae has been investigated to study the occurrence of metabolic channelling in S. cerevisiae (Sumegi et al., 1990). Since knowledge of the actual pathway of propionate metabolism by $S$. cerevisiae is essential for a correct interpretation of their results, we studied the activity of a number of key enzymes of propionate-degrading pathways in cell-free extracts.

\section{METHODS}

Micro-organisms and maintenance. Saccharomyces cerevisiae CBS 8066 was obtained from the Centraal Bureau voor Schimmelcultures, Delft, the Netherlands, and was maintained by monthly subculture on malt agar slopes. Stock cultures were stored at $4{ }^{\circ} \mathrm{C}$. Paracoccus denitrificans LMD 22.21 was obtained from the Culture Collection of the Laboratory of Microbiology and Enzymology, Delft, the Netherlands, and maintained on peptone agar slants.

Chemostat cultivation. Aerobic chemostat cultivation of S. cerevisiae was performed at $30^{\circ} \mathrm{C}$ in 1 litre Applikon laboratory fermenters at a stirring speed of 750 r.p.m. and at a dilution rate of $0.05 \mathrm{~h}^{-1}$. The cultures were flushed with air $\left(0.51 \mathrm{~min}^{-1}\right)$. The dissolved-oxygen concentration in the cultures was above $80 \%$ of air saturation. The culture $\mathrm{pH}$ was controlled at 5.0 by automatic addition of $2 \mathrm{M} \mathrm{KOH}$. The condenser was connected to a cryostat and cooled at $2{ }^{\circ} \mathrm{C}$. The working volume of the culture was kept at 1.0 litre by a peristaltic effluent pump coupled to an Applikon level controller. Biomass concentrations in samples taken directly from the culture differed by less than $1 \%$ from biomass concentrations in the culture effluent. The mineral medium, supplemented with vitamins, was prepared according to Verduyn et al. (1992). Vitamins were filtersterilized and added after autoclaving the mineral medium. Glucose was sterilized separately at $110^{\circ} \mathrm{C}$. Pure propionic acid was added to sterile reservoir media without prior sterilization. 
Cultures were checked for the occurrence of oscillations (Sonnleitner, 1991) by continuous registration of the dissolvedoxygen concentration. All data presented were derived from steady-state cultures without detectable oscillations. The purity of chemostat cultures was routinely checked by phase contrast microscopy at $1000 \times$ magnification and by plating on complex media.

Cultivation of Paracoccus denitrificans. $P$. denitrificans was grown at $30^{\circ} \mathrm{C}$ in shake flask cultures on the mineral medium described by Van Schie et al. (1984), supplemented with $20 \mathrm{mM}$ sodium propionate.

Determination of culture dry weight. Dry weights of culture samples were determined using a microwave oven and $0.45 \mu \mathrm{m}$ membrane filters as described by Postma et al. (1989). Parallel samples varied by less than $1 \%$.

Analysis of substrates and metabolites. Glucose was assayed with the Boehringer GOD-PAP glucose kit (no. 676543). Propionate was determined by HPLC on an HPX-87H column $(300 \times 7.8 \mathrm{~mm}$, Bio-Rad $)$ at room temperature. Detection was by means of a Waters $441 \mathrm{UV}$ detector at $214 \mathrm{~nm}$, coupled to a Waters 741 data module. Peak areas were linearly proportional to concentrations. The detection limit for propionate was approximately $50 \mu \mathrm{M}$. The same HPLC set-up was used for detection of organic acids in culture supernatants and for the detection of glycerol and ethanol. In the latter case, the same column was used, but detection was by means of an Erma 7515 A refractive index detector.

Protein determination. Protein concentrations in cell-free extracts were estimated by the Lowry method. The protein content of culture samples was estimated by a modified biuret method (Verduyn et al., 1992). In both assays, bovine serum albumin (fatty-acid-free, Sigma) was used as a standard.

Preparation of cell-free extracts. Samples of steady-state chemostat cultures were harvested by centrifugation, washed twice with $10 \mathrm{mM}$ potassium phosphate buffer $(\mathrm{pH} 7.5)$ containing $2 \mathrm{mM}$ EDTA, concentrated to a final concentration of $10-15 \mathrm{mg} \mathrm{dry}$ wt $\mathrm{ml}^{-1}$ and stored at $-40^{\circ} \mathrm{C}$ in $5 \mathrm{ml}$ aliquots. Before preparation of extracts, the samples were thawed at room temperature, washed twice and resuspended in ice-cold

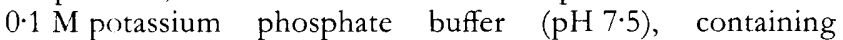
$1 \mathrm{mM}$ dithiothreitol and $2 \mathrm{mM} \mathrm{MgCl}$. Extracts were prepared by sonication with $0.1 \mathrm{~mm}$ diameter glass beads at $0^{\circ} \mathrm{C}$ for $2 \mathrm{~min}$ at $0.5 \mathrm{~min}$ intervals with an MSE sonicator (150 $\mathrm{W}$ output, $8 \mu \mathrm{m}$ peak-to-peak amplitude). Unbroken cells and debris were removed by centrifugation at $4{ }^{\circ} \mathrm{C}$ $(20 \mathrm{~min}$ at $75000 \mathrm{~g}$ ). The clear supernatant, typically containing 1-3 mg protein $\mathrm{ml}^{-\mathbf{1}}$, was used as the cell-free extract.

Enzyme assays. Enzyme activities were assayed immediately after preparation of the cell-free extracts. All enzyme assays were carried out at $30^{\circ} \mathrm{C}$. In all activity measurements, the reaction rates were linearly proportional to the amount of cell-free extract added.

Citrate synthase (EC 4.1.3.7). The reaction mixture $(1 \mathrm{ml})$ contained: Tris/ $\mathrm{HCl}$ buffer $(\mathrm{pH} 8 \cdot 0), 100 \mu \mathrm{mol}$; acetyl-CoA, $0.05 \mu \mathrm{mol}$; 5,5'-dithiobis(2-nitrobenzoic acid) (DTNB), $0 \cdot 1 \mu \mathrm{mol}$; and cell-free extract. The reaction was started by the addition of $0.2 \mu \mathrm{mol}$ oxaloacetate. Release of CoA was monitored at $412 \mathrm{~nm}$ (Srere, 1969) and activities were calculated using an extinction coefficient of $13.6 \mathrm{mM}^{-1} \mathrm{~cm}^{-1}$.

2-Methylcitrate synthase. The reaction mixture $(1 \mathrm{ml})$ contained; Tris $/ \mathrm{HCl}$ buffer $(\mathrm{pH} \mathrm{8.0)}, 100 \mu \mathrm{mol}$; propionyl-CoA,
$0 \cdot 1 \mu \mathrm{mol} ; \mathrm{DTNB}, 0 \cdot 1 \mu \mathrm{mol}$; and cell-free extract. The reaction was started by the addition of $0 \cdot 2 \mu \mathrm{mol}$ oxaloacetate and monitored as described for citrate synthase.

Acetyl-COA synthetase (EC 6 . 2.1.1) and propionyl-COA synthetase. Enzyme activity was determined by means of a discontinuous asay, measuring the consumption of $\mathrm{CoA}$ (Webster, 1969). The reaction mixture $(1 \mathrm{ml})$ contained: Tris/ $\mathrm{HCl}$ buffer ( $\mathrm{pH} \mathrm{8.0)}, 100 \mu \mathrm{mol} ; \mathrm{MgCl}_{2}, 5 \mu \mathrm{mol} ; \mathrm{NiCl}_{2}, 0.02 \mu \mathrm{mol}$; ATP , $2.5 \mu \mathrm{mol}$; $\mathrm{CoA}, 1 \mu \mathrm{mol}$; and cell-free extract. The reaction was started by the addition of either $2 \mu \mathrm{mol}$ sodium acetate or $20 \mu \mathrm{mol}$ sodium propionate. At appropriate time intervals, $50 \mu \mathrm{l}$ samples were withdrawn from the reaction mixture and added to $950 \mu \mathrm{l}$ of a solution containing $100 \mu \mathrm{mol}$ Tris/ $\mathrm{HCl}$ buffer $(\mathrm{pH} 8.0)$, and $0.2 \mu \mathrm{mol}$ DTNB. Decrease of the CoA concentration was linear with time and proportional to the amount of cell-free extract added.

Propionyl-CoA carboxylase (EC 6 4 4. 3.1). Enzyme activity was measured as incorporation of $\left[{ }^{14} \mathrm{C}\right]$ bicarbonate (Kaziro, 1969). The reaction mixture $(1 \mathrm{ml})$ contained: Tris $/ \mathrm{HCl}$ buffer (pH 8.0), $100 \mu \mathrm{mol} ; \mathrm{MgCl}_{2}, 5 \mu \mathrm{mol}$; reduced glutathione, $2 \mu \mathrm{mol}$; ATP, $2.5 \mu \mathrm{mol}$; $\mathrm{NaH}^{14} \mathrm{CO}_{3}\left(37 \mathrm{GBq} \mathrm{mol}^{-1}\right), 2.5 \mu \mathrm{mol}$; and cell-free extract. The reaction was started by the addition of $0 \cdot 1 \mu \mathrm{mol}$ propionyl-CoA. At appropriate time intervals, $50 \mu \mathrm{l}$ samples were withdrawn from the mixture and added to $200 \mu \mathrm{l}$ acetic acid, to expel non-bound $\mathrm{CO}_{2}$. After evaporation of acetic acid at $60^{\circ} \mathrm{C}$, the pellet was dissolved in $0.5 \mathrm{ml}$ water. Beckman Ready-Safe scintillation cocktail $(5 \mathrm{ml})$ was added and radioactivity was measured in a Beckman LS 3801 scintillation counter. Cell-free extracts of propionate-grown Paracoccus denitrificans LMD 22.21 were included as a positive control, since this organism is known to metabolize propionate via methylmalonyl-CoA (Smith \& Kornberg, 1967).

Chemicals. Sodium $\left[{ }^{14} \mathrm{C}\right]$ bicarbonate $\left(2 \cdot 11 \mathrm{TBq} \mathrm{mol}^{-1}\right)$ was obtained from Amersham. All other chemicals were reagent grade and obtained from commercial sources.

\section{RESULTS AND DISCUSSION}

\section{Utilization of propionate by S. cerevisiae}

When $10 \mathrm{mM}$ propionic acid was added to the reservoir media of glucose-limited chemostat cultures of S. cerevisiae, the residual concentration of propionate in the culture remained below $100 \mu \mathrm{M}$. At this low residual concentration, propionate does not have a significant uncoupling effect on the energy metabolism of $S$. cerevisiae (Verduyn et al., 1990a). Instead, the consumption of propionic acid by the cultures coincided with an increase of the biomass concentration (Fig. 2). A further increase of the reservoir concentration of propionic acid led to a linear increase of the steady-state biomas concentration (Fig. 2), with the residual propionate concentration remaining below $100 \mu \mathrm{M}$. Also, the protein content of the cultures increased linearly over the range of propionate concentrations tested (Fig. 2).

Only small increases of the propionic acid concentration in the reservoir media could be made in the mixedsubstrate cultures: increases of over $10 \mathrm{mM}$ propionic acid at a time resulted in wash-out. Attempts to switch cultures growing on $14 \mathrm{mM}\left(2.5 \mathrm{~g} \mathrm{l}^{-1}\right)$ glucose and $50 \mathrm{mM}$ propionic acid to growth on $40 \mathrm{mM}$ propionic acid as the sole source of carbon and energy were 


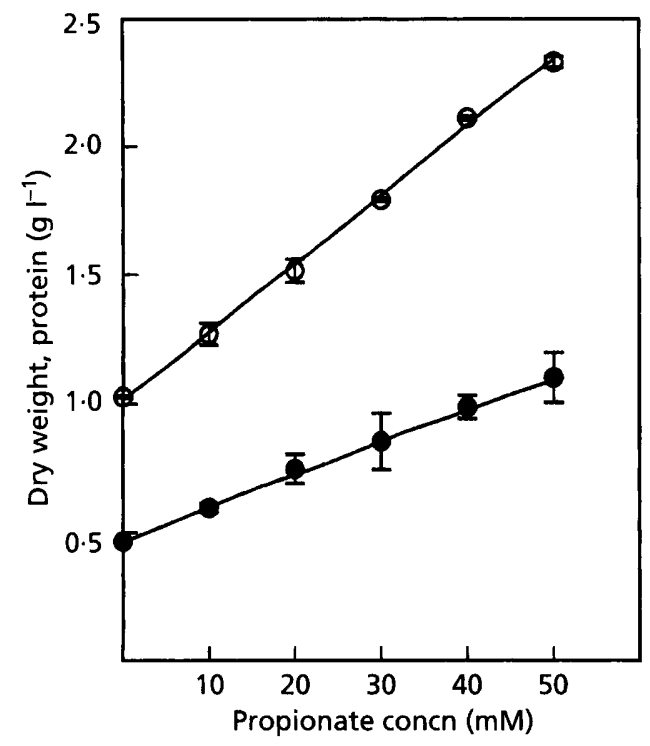

Fig. 2. Growth of $S$. cerevisiae CBS 8066 on glucose $\left(2.5 \mathrm{~g} \mathrm{I}^{-1}\right)$ with increasing concentrations of propionic acid in the reservoir media. Growth conditions: $D=0.05 \mathrm{~h}^{-1}, \mathrm{pH} 5 \cdot 0, T=30^{\circ} \mathrm{C}$, d.o.t. $>80 \%$ air saturation. $O$, Culture dry weight; 0 , protein concentration. Data are presented as means $\pm S D$ of two independent chemostat runs.

unsuccessful: the cultures washed out. These experiments do not necessarily imply that $S$. cerevisiae is unable to grow on propionate as a sole carbon source. Propionate is toxic to $S$. cerevisiae, and it may well be that propionate-limited steady-state cultures can be attained by a more careful manipulation of the composition of the reservoir medium or at a lower dilution rate.

In addition to the effects on biomass yield, addition of propionic acid to the reservoir media also affected the morphology of the yeast cells. Glucose-limited cultures mainly contained free cells and cells with one bud. In mixed-substrate cultures, a substantial fraction of the buds did not dissociate from the mother cells, leading to the formation of small aggregates (typically 3-8 cells). The occurrence of these aggregates did not influence the removal of cells with the culture effluent: biomass concentrations in samples taken directly from the culture and in samples taken from the effluent line differed by less than $1 \%$. Electron microscopy of thin sections revealed an increase of the mitochondrial volume with increasing propionate flux. Proliferation of peroxisomes, as reported for propionate-grown Candida tropicalis (Ueda et al., 1983, 1989), was not observed (data not shown).

\section{Enzymology of propionate metabolism}

To investigate which pathway of propionate metabolism is operating in S. cerevisiae, the activity of three potential key enzymes was assayed in cell-free extracts. PropionylCoA synthetase catalyses the activation of propionate (Fig. 1). Propionyl-CoA carboxylase and 2-methylcitrate synthase are key enzymes of the methylmalonyl-CoA pathway and the 2-methylcitric acid cycle, respectively (Fig. 1).

Acetyl-CoA synthetase (EC 6.2.1.1) from S. cerevisiae can also catalyse the formation of propionyl-CoA from propionate and coenzyme A (Jones \& Lipmann, 1955). In cell-free extracts, activities with both substrates could be detected at the various ratios of propionic acid to glucose in the reservoir media (Fig. 3). The propionyl-CoA synthetase activities measured in cell-free extracts were sufficient to account for the flux of propionate in the chemostat cultures (Fig. 3). The ratio between acetyl-CoA synthetase activity and propionyl-CoA synthetase activity $(3 \cdot 6 \pm 1 \cdot 2)$ did not exhibit a clear correlation with the composition of the reservoir medium. A similar ratio (3.5) was observed in ethanol-limited chemostat cultures. These observations are consistent with catalysis of the initial step of propionate metabolism in $S$. cerevisiae by acetyl-CoA synthetase. Acetyl-CoA synthetase levels in aerobic, glucose-limited S. cerevisiae cultures decrease with increasing growth rate (Postma et al., 1989). This may explain the observation that, at a dilution rate of $0.30 \mathrm{~h}^{-1}$, propionate was not consumed by aerobic, glucose-limited chemostat cultures of $S$. cerevisiae CBS 8066 (Postma et al., 1989).

The initial step in propionyl-CoA metabolism via the methylmalonyl-CoA pathway is the carboxylation of propionyl-CoA, yielding the key intermediate of this pathway, methylmalonyl-CoA (Fig. 1a). This reaction is catalysed by the enzyme propionyl-CoA carboxylase (EC 6.4.1.3). Activities of this enzyme in cell-free extracts of all chemostat cultures were below $0.5 \mathrm{nmol} \mathrm{min}{ }^{-1} \mathrm{mg}^{-1}$. This is over 100 -fold lower than the maximum observed propionate consumption rates in the chemostat cultures, which makes it unlikely that the enzyme plays a significant role in propionate metabolism in the cultures. In control experiments, high activities of propionyl-CoA carboxylase $\left(30-50 \mathrm{nmol} \mathrm{min} \mathrm{mg}^{-1} \mathrm{mg}^{-1}\right.$ were observed in cell-free extracts of propionate-grown Paracoccus denitrificans, indicating that the low activities observed in the $S$. cerevisiae extracts were not due to errors in the assay procedure.

The 2-methylcitrate pathway of propionate metabolism is initiated by the condensation of propionyl-CoA and oxaloacetate, yielding 2-methylcitrate (Tabuchi \& Uchiyama, 1975). The activity of the enzyme responsible, 2 methylcitrate synthase, increased with increasing propionate-to-glucose ratios in the reservoir media of the chemostat cultures and was paralleled by an increase of the citrate synthase activity (Fig. 4). When suboptimal concentrations of propionyl-CoA were added to the enzyme assays, the amount of $\mathrm{CoA}$ released was equal to the amount of propionyl-CoA added (data not shown), indicating that the observed activities were not due to contamination of the propionyl-CoA with acetyl-CoA. Apparently, the 2-methylcitrate synthase reaction is catalysed by the $S$. cerevisiae citrate synthase. Likewise, the Yarrowia lipolytica 2-methylcitrate synthase exhibits this dual substrate specificity (Uchiyama \& Tabuchi, 1976). 


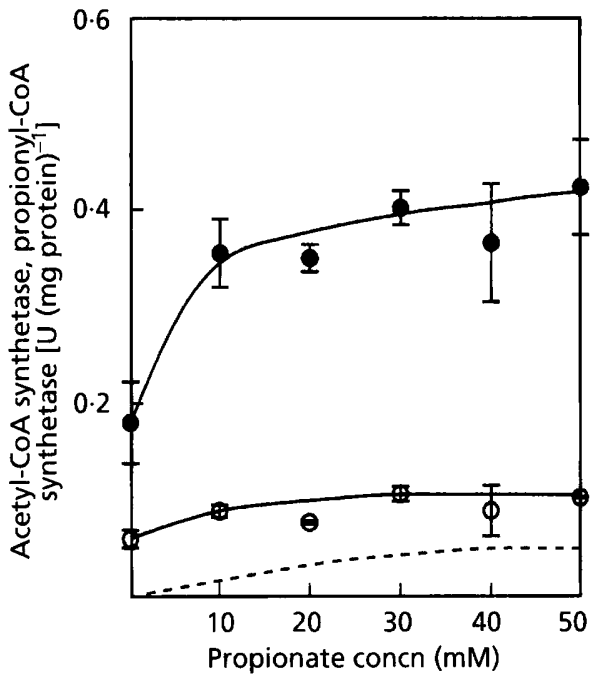

Fig. 3

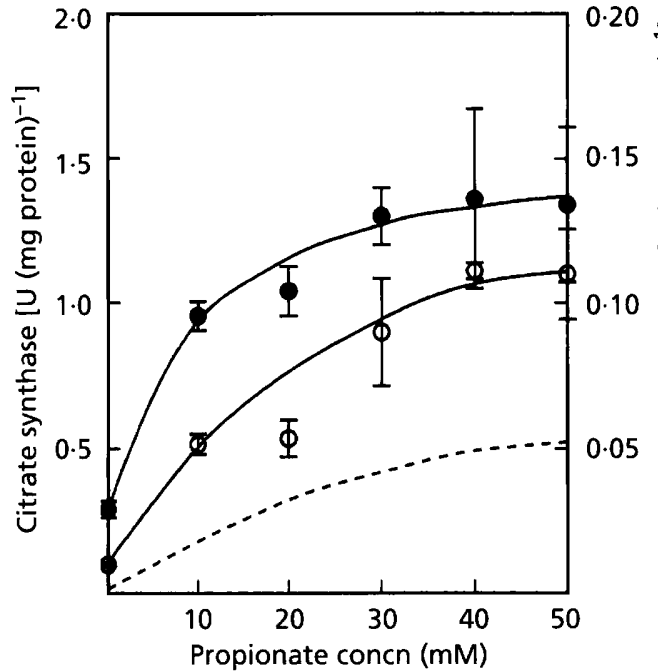

Fig. 4

Fig. 3. Activities of acetyl-COA synthetase $(\bullet)$ and propionyl-CoA synthetase $(O)$ in cell-free extracts of $S$. cerevisiae CBS 8066 pregrown in glucose-limited chemostat cultures supplemented with increasing concentrations of propionic acid in the reservoir medium (growth conditions as in legends to Fig. 2). Data are presented as means \pm SD of two independent chemostat runs. The dashed line indicates estimated in vivo rates of propionate consumption in the chemostat cultures, calculated on the basis of a soluble-protein content of yeast biomass of $33 \%$ (Postma et al., 1989).

Fig. 4. Activities of citrate synthase $(O)$ and 2-methylcitrate synthase $(O)$ in cell-free extracts of $S$. cerevisiae pregrown in glucose-limited chemostat cultures supplemented with increasing concentrations of propionic acid in the reservoir medium (growth conditions as in legends to Fig. 2). Data are presented as means \pm SD of two independent chemostat runs. The dashed line (plotted against the right ordinate) indicates the estimated in vivo rates of propionate consumption in the chemostat cultures (see legend to Fig. 3).

The 2-methylcitrate synthase activity was sufficient to account for the fluxes of propionate observed in the chemostat cultures of $S$. cerevisiae growing on mixtures of glucose and propionate (Fig. 4).

The above data suggest that instead of the methylmalonylCoA pathway, the 2-methylcitrate pathway is the major route of propionate metabolism in $S$. cerevisiae. The net result of propionate conversion via this pathway is its oxidation to pyruvate. Although toxic at high concentrations, propionate is not an unnatural product in the intermediary metabolism of yeasts. For example, propionyl-CoA is an intermediate in the degradation of isoleucine. Recently, it has been suggested that the 2methylcitrate pathway may be responsible for the formation of succinate from isoleucine by $S$. cerevisiae during beer fermentation (Shindo et al., 1993).

\section{Metabolic channelling}

It has been suggested in the literature that the enzymes of the TCA cycle should not be considered to be soluble in the mitochondrial matrix but, instead, form a supramolecular complex bound to the inner surface of the mitochond rial inner membrane, called a metabolon (Srere, 1985, 1990). Organization of enzymes in metabolons could lead to a phenomenon called 'channelling', i.e. the transfer of intermediates between the enzymes of a metabolic pathway occurs without equilibration with the bulk water phase.

The distribution of ${ }^{13} \mathrm{C}$ in alanine after incubation of S. cerevisiae cells with $\left[3-{ }^{13} \mathrm{C}\right]$-propionate has been used to study channelling of TCA cycle intermediates in yeast mitochondria (Sumegi et al., 1990). For interpretation of their experimental data, Sumegi et al. (1990) assumed that metabolism of propionate by $S$. cerevisiae occurs via the methylmalonyl-CoA pathway (Fig. 1a). Starting with [3${ }^{13} \mathrm{C}$ ]propionate, this pathway results in the formation of $\left[2-{ }^{13} \mathrm{C}\right]$ succinyl-CoA. Upon removal of the CoA moiety, succinate is formed. Since this is a symmetrical molecule, no enrichment of ${ }^{13} \mathrm{C}$ in either $\mathrm{C}-2$ or $\mathrm{C}-3$ of alanine is expected when transfer of TCA cycle intermediates between the enzymes of the cycle occurs by free diffusion through the bulk liquid phase of the mitochondrial matrix. However, Sumegi et al. (1990) did observe a significant enrichment of ${ }^{13} \mathrm{C}$ label in $\mathrm{C}-3$ of alanine. This was interpreted as evidence for channelling of TCA cycle intermediates in $S$. cerevisiae mitochondria (Sumegi et al., 1990; Srere, 1990; Evans et al., 1993).

The data presented in this paper suggest that propionate metabolism in $S$. cerevisiae occurs predominantly via the 2 methylcitrate pathway. Conversion of $\left[3-{ }^{13} \mathrm{C}\right]$ propionate into pyruvate via this linear pathway should yield exclusively $\left[3-{ }^{13} \mathrm{C}\right]$ pyruvate (Fig. 1b). Only when pyruvate re-enters the citric acid cycle may some label be scrambled 
and appear at C-2 of alanine as well. Accordingly, a major involvement of the 2-methylcitrate pathway is fully compatible with the labelling pattern of alanine formed from $\left[3-{ }^{13} \mathrm{C}\right]$ propionate as observed by Sumegi et al. (1990). Consequently, their data cannot be interpreted as proof for the occurrence of metabolic channelling of TCA cycle intermediates in S. cerevisiae or the existence of a TCA cycle metabolon in this yeast.

\section{ACKNOWLEDGEMENT}

We thank Wilma Batenburg-van der Vegte for performing electron microscopy.

\section{REFERENCES}

Evans, C. T., Sumegi, B., Srere, P. A., Sherry, A. D. \& Malloy, C. R. (1993). $\left[{ }^{13} \mathrm{C}\right]$ Propionate oxidation in wild-type and citrate synthase mutant Escherichia coli: evidence for multiple pathways of propionate utilization. Biochem J 291, 927-932.

Jones, M. E. \& Lipmann, F. (1955). Aceto-CoA-kinase. Methods Enzymol 1, 585-591.

Kaziro, Y. (1969). Crystalline propionyl-CoA carboxylase from pig heart. Methods Enzymol 13, 181-190.

Postma, E., Verduyn, C., Scheffers, W. A. \& van Dijken, J. P. (1989). Enzymic analysis of the Crabtree effect in glucose-limited chemostat cultures of Saccharomyces cerevisiae. Appl Environ Microbiol 53, 468-477.

Schneider, Z. \& Stroinski, A. (1987). Comprehensive $B_{12}$. Hawthorne, USA: Walter de Gruyter.

Shindo, S., Sahara, H. \& Koshino, S. (1993). Relationship of production of succinic acid and methyl citric acid pathway during alcohol fermentation with immobilized yeast. Biotecbnol Lett 15, $51-56$.

Smith, J. \& Kornberg, H. L. (1967). The utilization of propionate by Micrococcus denitrificans. J Gen Microbiol 47, 175-180.

Sonnleitner, B. (1991). Dynamics of yeast metabolism and regulation. Bioprocess Eng 6, 187-193.

Srere, P. A. (1969). Citrate synthase. Methods Enzymol 13, 3-11.

Srere, P. A. (1985). The metabolon. Trends Biochem Sci 10, 109-110.

Srere, P. A. (1990). Citric acid cycle redux. Trends Biochem $S_{c i} \mathbf{1 5}$, $411-412$.
Sumegi, B., Sherry, A. D. \& Malloy, C. R. (1990). Channeling of TCA cycle intermediates in cultured Saccharomyces cerevisiae. Biochemistry 29, 9106-9110.

Tabuchi, T. \& Uchiyama, H. (1975). Methylcitrate condensing and methylisocitrate cleaving enzymes; evidence for the pathway of oxidation of propionyl-CoA to pyruvate via $\mathrm{C}_{7}$-tricarboxylic acids. Agric Biol Chem 39, 2035-2042.

Uchiyama, H. \& Tabuchi, T. (1976). Properties of methylcitrate synthase from Candida lipolytica. Agric Biol Chem 40, 1411-1418.

Uchiyama, H., Ando, M., Toyonaka, Y. \& Tabuchi, T. (1982). Subcellular localization of the methylcitric acid cycle enzymes in propionate metabolism of Yarrowia lipolytica. Eur J Biochem 125, 523-527.

Ueda, M., Okada, H., Tanaka, A., Osumi, M. \& Fukui, S. (1983). Induction and subcellular localization of enzymes participating in propionate metabolism in Candida tropicalis. Arch Microbiol 136, 169-176.

Ueda, M., Mozaffar, S., Atomi, H., Osumi, M. \& Tanaka, A. (1989). Characterization of peroxisomes in an $n$-alkane-utilizing yeast, Candida tropicalis, grown on glucose and propionate. J Ferment Bioeng 68, 411-416.

van Schie, B. J., van Dijken, J. P. \& Scheffers, W. A. (1984). Noncoordinated synthesis of glucose dehydrogenase and its prosthetic group PQQ in Acinetobacter and Pseudomonas species. FEMS Microbiol Lett 24, 133-138.

Verduyn, C., Postma, E., Scheffers, W. A. \& van Dijken, J.P. (1990a). Physiology of Saccharomyces cerevisiae in anaerobic glucoselimited chemostat cultures. J Gen Microbiol 136, 395-403.

Verduyn, C., Postma, E., Scheffers, W. A. \& van Dijken, J.P. (1990b). Energetics of Saccharomyces cerevisiae in anaerobic glucoselimited chemostat cultures. J Gen Microbiol 136, 405-412.

Verduyn, C., Postma, E., Scheffers, W. A. \& van Dijken, J.P. (1992). Effect of benzoic acid on metabolic fluxes in yeasts: a continuous culture study on the regulation of respiration and alcoholic fermentation. Yeast 8, 501-517.

Webster, L. T. (1969). Acetyl-CoA synthetase. Methods Enzymol 13, 375-381.

Wegener, W. S., Reeves, H. C., Rabin, R. \& Ajl, S. J. (1968). Alternate pathways of metabolism of short-chain fatty acids. Bacteriol Rev 32, 1-26.

Received 19 July 1993; revised 12 October 1993; accepted 26 October 1993 